\title{
In vitro apoptosis triggering of human breast cancer T47D cell line by lyophilised camel milk
}

Sidgi Syed Anwer Hasson*1, Mohamed Ahmed Idris1, Ali Abdullah Al-Jabri1, Talal A. Sallam2

1Department of Microbiology and Immunology, College of Medicine and Health Sciences, Sultan Qaboos University, Oman.

2Department of Medical Microbiology, Faculty of Medicine, Al-Baha University, Kingdom of Saudi Arabia.

Corresponding Author

Dr. Sidgi Hasson,

Dept. of Microbiology \& Immunology, Rm. 1028,

College of Medicine \& Health Sciences,

Sultan Qaboos University,

P.O. Box 35, Al-Khod,

Postal Code 123,

Sultanate of Oman,

Phone: (968) 24143549, 
Proc. of the Third Intl. Conf. on Advances in Bio-Informatics and Environmental Engineering - ICABEE 2015

Copyright $\odot$ Institute of Research Engineers and Doctors, USA .All rights reserved.

ISBN: 978-1-63248-078-1 doi: 10.15224/ 978-1-63248-078-1-107

\begin{abstract}
Breast cancer is a worldwide health disquiet and is the second leading cause of cancer death among women. In Oman, it is the most well-known malignancy in women, with an incidence rate of 15.6 per 100,000 of the Omani females. Several anticancer remedies have been found from natural products in the past and the search is continuing to add more examples. Cytotoxic from natural compounds may have a considerable aspect in cancer therapy either in potentiating the impact of chemotherapy or curtailment its harmful effects. As of late, a couple studies have reported the benefit of utilizing crude camel milk as a part of treating a few types of cancer. In any case, no sufficient data are at present accessible on the potential of lyophilised camel's milk for the triggering of apoptosis and oxidative stress linked with human breast cancer. The present study intended to address the potential role of the lyophilised camel's milk in inducing proliferation suppression of T47D and HEp-2 cells in contrast with the non-cancer HCC1937 BL cell line. Lyophilized camel's milk principally suppressed T47D cells growth and proliferation through the induction of either the intrinsic and extrinsic apoptotic pathways demonstrated by both caspase-3 mRNA and its activity level, and the impelling of death receptors in T47D but not the HEp-2 cell line. In addition to that lyophilised camel milk found to promotes the expression of oxidative stress markers as well as the production of both the heme- oxygenase- 1 and reactive oxygen in T47D cells. The increase in the mRNA levels of caspase- 3 found to be totally blocked by actinomycin $D$, a transcriptional inhibitor. This proposes that lyophilized camel milk increased newly synthesized RNA. Interestingly, the lyophilized camel's milk significantly $(p<0.005)$ repressed the growth of HEp- 2 cells and T47D cells after treatment for 72 hours while 24 hours treatment suppressed T47D cells alone. This finding suggests that the lyophilised camel milk might influence apoptosis through initiation of an alternative apoptotic pathway (s).
\end{abstract}

KEYWORDS: Breast cancer, T47D cell, camel milk, anticancer activity, zymography, Oman 